\title{
Predicting lymph node metastasis in pT1 colorectal cancer: a systematic review of risk factors providing rationale for therapy decisions
}

Authors

Institutions
Steven L. Bosch ${ }^{1}$, Steven Teerenstra ${ }^{2}$, Johannes H. W. de Wilt ${ }^{3}$, Chris Cunningham ${ }^{4}$, Iris D. Nagtegaal ${ }^{1}$

Institutions are listed at the end of article. submitted

10. October 2012

accepted after revision

3. May 2013

\section{Bibliography}

DOI http://dx.doi.org/

10.1055/s-0033-1344238

Published online: 2013

Endoscopy

(c) Georg Thieme Verlag KG

Stuttgart · New York

ISSN 0013-726X

\section{Corresponding author}

\section{Steven L. Bosch, MD}

Department of Pathology 824

Radboud University Nijmegen Medical Center

PO Box 9101, 6500 HB

Nijmegen

The Netherlands

Fax: +31-24-3668750

s.bosch@pathol.umcn.nl
Background and study aim: Population screening for colorectal cancer (CRC) is expected to increase the number of pT1 CRCs. Local excision is an attractive treatment option, but is only oncologically safe in the absence of lymph node metastasis (LNM). A systematic review of the predictive value of pathological risk factors for LNM in PT1 CRC was conducted to provide data for an evidencebased decision regarding follow-up or radical surgery after local excision.

Methods: PubMed was searched for reports on predictors of LNM in PT1 CRC. Published papers written in English and containing at least 50 patients were included. Meta-analyses were performed using Review Manager 5.1.

Results: A total of 17 studies were included involving a total of 3621 patients with available nodal status. The strongest independent predictors of LNM were lymphatic invasion (relative risk [RR]

\section{Introduction}

$\nabla$

With the widespread introduction of population screening for colorectal cancer (CRC), the number of early CRCs is expected to increase [1-6]. In the past, approximately $25 \%$ of CRC cases presented with early disease (stage I) in which the primary tumor is limited to the submucosa (T1 tumors) or the bowel wall (T2 tumors). Local excision is an attractive option for early disease in both colon and rectal cancer, as it is associated with considerably less surgery-related morbidity and almost no postoperative mortality compared with colectomy and total mesorectal excision (TME) [7-13], for which mortality rates of $1.9 \%-6.5 \%$ (rectum) and $3.2 \%-9.8 \%$ (colon) have been reported [14, 15].

Clinically, local treatment is especially relevant for rectal cancer, as the consequences of TME surgery, which often results in a colostomy, sexual and urinary dysfunction, and complaints of soiling and fecal incontinence, are greater than for
5.2, 95\% confidence interval $[\mathrm{CI}] 4.0-6.8)$, submucosal invasion $\geq 1 \mathrm{~mm}$ (RR 5.2 , 95\%CI 1.8 15.4), budding (RR 5.1, 95\%CI 3.6-7.3), and poor histological differentiation (RR 4.8, 95\%CI 3.36.9). Limitations of the study were: results could not be stratified according to location in the colon or rectum; very early tumors removed by polypectomy without surgical resection were not included in the meta-analysis; and included studies were primarily from Asian countries and results therefore need to be verified in Western populations.

Conclusion: The absence of lymphatic invasion, budding, submucosal invasion $\geq 1 \mathrm{~mm}$, and poor histological differentiation were each associated with low risk of LNM. Risk stratification models integrating these factors need to be investigated further.

colectomy [16-20]. Moreover, the removal of tumors in the rectum, using transanal endoscopic microsurgery (TEM) and endoscopic submucosal dissection (ESD), is more effective than in the colon, where ESD is technically difficult and where mainly polypectomy and endoscopic mucosal resection (EMR) are used $[13,21]$. However, there is a considerable move towards organ-preserving surgery for colon cancer as well, as techniques for local treatment in the colon are improving [22].

Local excision is generally reserved for T1 disease, as adequate removal of T2 tumors using ESD or EMR in the colon is not feasible [23], and for rectal cancer local treatment of T2 lesions is reported to result in unacceptably high local recurrence rates and a lower survival compared with radical resection $[10,24]$.

Currently, patient-related factors such as age and co-morbidity are of primary importance when deciding whether or not to perform a radical resection for early CRC. For fit patients with T1 
CRC, the curative intent is absolute and a local excision can only be oncologically safe in the absence of lymph node metastasis (LNM). The overall incidence of LNM in T1 tumors is between $8 \%$ and $16 \%$ [25-28], and several pathological features of the primary tumor, such as poor differentiation, lymphatic or vascular invasion, and submucosal invasion depth, have been associated with its presence [29-32].

Patient selection through careful histological analysis of local excision specimens can therefore be very useful to avoid over- and under-treatment. For rectal cancer, some of the characteristics mentioned above are already included in national guidelines as indicators of high risk lesions necessitating additional radical surgery (e.g. American [National Cancer Institute], British, Japanese and Dutch national guidelines [33-36]).

However, despite the importance of high quality discrimination between low and high risk T1 cases, an adequate overview of the literature that quantifies the influence of the individual risk factors, is lacking. Although the described differences regarding treatment options and associated morbidity suggest that the clinical relevance is currently greatest for rectal cancer, risk stratification is valuable for pT1 colon cancer as well.

Unfortunately, a separate analysis is not feasible, as studies describing nodal involvement exclusively for pT1 rectal cancer are very scarce. Therefore, the current study provides a systematic review of the risk factors for the presence of LNM in PT1 CRC including meta-analyses where appropriate.

\section{Methods}

$\nabla$

\section{Search strategy and selection criteria}

A comprehensive literature search was performed using the PubMed database from inception to 25 May 2011. The keywords used were "lymph nodes," "lymph node metastasis," "TEM," and "T1" combined with "colorectal cancer." Additional searches were performed using manual cross-referencing. Only published studies written in English and with at least 50 patients were included. Reports describing use of neo-adjuvant therapy (ypT) were not included. Radical resection was required to obtain a reliable lymph node status. The percentage or number of patients with nodal involvement, specified for presence and absence of a specific risk factor, was required. Data from pT1 patients had to be reported separately. In case of possible overlap of data due to duplicate publications, only the article with the largest sample size was included.

\section{Measuring submucosal invasion depth}

Various methods of dividing patients into a low or high risk group based on submucosal invasion depth are described in the literature. For sessile lesions a qualitative assessment according to Kudo et al. (1993) [37] is commonly used (sm1, $\mathrm{sm} 2$, and sm3: invasion into the most superficial, intermediate, and deepest $1 / 3$ of the submucosa, respectively). This has been modified slightly into a semi-quantitative system by Kikuchi et al. (1995) [32] (sm1: invasion up to 0.2-0.3 mm; sm2: intermediate invasion; sm3: invasion near the muscularis propria). A third method quantifies invasion depth ( $\mathrm{sm} 1$ : up to $0.5 \mathrm{~mm}$; sm2: $0.5-1.0 \mathrm{~mm}$; sm3: beyond $1.0 \mathrm{~mm}$ ) [38]. Alternatively, the invasion depth is measured and a cut-off value is defined to distinguish between superficial and deep submucosal invasion. For the purpose of performing the current meta-analysis, the studies were divided into two groups: one group included stud- ies using a quantitative invasion depth and the other included studies using qualitative or semi-quantitative invasion depth.

\section{Statistical analysis}

Data were extracted and analyzed by a single investigator (S.L.B.). For all studies in the meta-analysis the frequencies of LNM per factor were available either from the text or from tables. Risk factors, incidence, and events from the individual studies were entered into Review Manager 5.1 (RevMan, Copenhagen: The Nordic Cochrane Centre, The Cochrane Collaboration, 2011). If a factor was reported in at least three studies with comparable methodology, a meta-analysis was performed to summarize its prognostic effect in terms of a relative risk (RR) with $95 \%$ confidence interval (CI). A random effects model with Mantel-Haenszel weighting was used. Heterogeneity was assessed using a $\chi^{2}$ test for heterogeneity, with a $P$ value of $<0.10$ taken to reflect the presence of significant heterogeneity. The $I^{2}$ statistic was calculated to quantify the degree of heterogeneity. Publication bias was assessed by inspection of the funnel plot by an experienced statistician (S.T.). Data presented as pooled estimates do not account for heterogeneity between studies and are reported for explorative purposes only.

\section{Results \\ $\nabla$}

\section{Study selection and inclusion}

The initial search returned a total of 43 studies ( $\bullet$ Fig. 1 ). Following review, 32 potentially relevant studies were identified as eligible of which 12 were excluded for duplicate series of patients, and $2[32,39]$ were excluded as they did not report separate data for patients with a radical resection and patients with local excision only.

In three studies [40-42], patients who underwent local excision only were reported separately and only the patients who received radical resection were included in the current analyses. Another study was excluded, because of a major discrepancy between data reported in text and tables [43]. Repeated attempts to contact the authors by email to clarify this issue were unsuccessful. Therefore, the data from this study were assumed to be unreliable.

The 17 studies selected for this review [28,38,40-42,44-55] included a total of 3782 patients with pT1 tumors. A total of 1561 patients had rectal carcinomas (41.3\%), 2080 patients had colon carcinomas (55.0\%), and in 141 patients no distinction was made between colon and rectal carcinomas (3.7\%). A total of 3621 patients had available nodal status after radical resection and only these patients were included in the meta-analysis. The median number of patients per study was 140 (range 65-865). - Table 1 outlines the characteristics of these studies. The incidence of LNM was $11.4 \%$ (414/3621). Of the included studies, 2 were performed prospectively and 15 were retrospective studies.

\section{Publication bias and heterogeneity}

Inspection of the funnel plots ( $\bullet$ Fig.e2, available online) did not reveal asymmetry, therefore there was no indication of publication bias. However, the funnel plot analysis was limited in many cases by the low number of studies. Forest plots ( Fig.e3-e8, available online) were checked for consistency of the effects. There was only quantitative heterogeneity. 


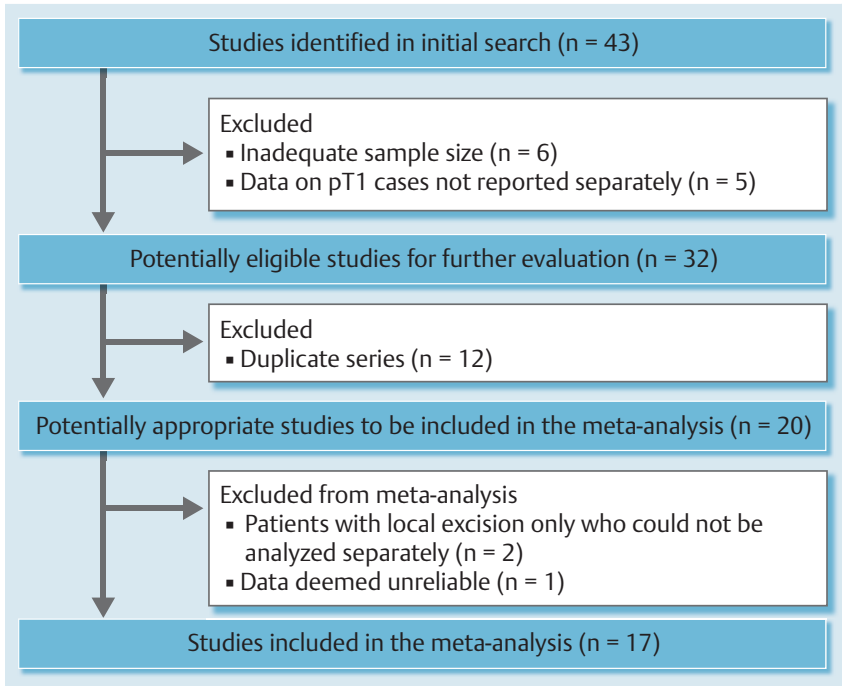

Fig. 1 Flow chart illustrating study selection process.

\section{Factors predicting LNM}

Factors that were investigated in at least three studies with comparable methodology were included in the meta-analyses. These factors are depicted in $\bullet$ Fig. 9 and include tumor location, lymphatic, vascular and lymphovascular invasion, submucosal invasion depth (based on sm-levels, cut-off value $1 \mathrm{~mm}$ and cut-off value $2 \mathrm{~mm}$ ), width of submucosal invasion (cut-off $5 \mathrm{~mm}$ ), histological differentiation grade (high grade vs. low grade), budding, and poor differentiation at the invasion front. Forest plots generated for the analysis of each individual risk factor are included in - Fig.e3-e8 (available online).
- Table 2 provides the data for the dichotomous risk factors with total number of patients available for each analysis, the calculated relative risks for presence of LNM, and the level of heterogeneity. The data for submucosal invasion depth based on the threetiered sm-levels (see Methods section) are shown in $\bullet$ Table 3. For all relevant factors the corresponding sensitivity, specificity, positive predictive value, and negative predictive value are included in Table 4. Table 5 (available online) shows the results of the multivariable analyses in the different studies. For submucosal invasion depth the methodology differs considerably between the studies.

\section{Lymphatic, vascular, and lymphovascular invasion}

Most studies included lymphatic or vascular (sometimes called venous) invasion either as separate variables or gathered under the heading of lymphovascular invasion. Definitions were not often provided, with the exception of Tsuruta et al. ([54]) and Wang et al. [55], where lymphatic invasion was defined as tumor cells in a space covered with endothelial cells in the absence of erythrocytes. Blood vessel invasion was defined as tumor within a space lined with either smooth muscle or endothelium with additional fibrin clots, erythrocytes or both without erythrocyte extravasation into the surrounding tissue [55]. Lymphovascular invasion was defined by Okabe et al. [51] as the presence of tumor cells within an epithelium-lined channel thought to represent either a lymphatic vessel or a blood vessel.

Additional staining techniques such as Victoria blue $[46,53]$ and Elastica von Gieson (EVG) $[44,45,54]$ or immunohistochemical stains such as D2-40 [45], LYVE-1 [46], vWF [46], and CD34 [42] were used by some authors.

Lymphatic invasion was the most powerful predictor of LNM emerging from the meta-analyses (RR 5.2 [95\%CI 4.0-6.8]). Multivariable analyses also provide solid evidence for an indepen-

Table 1 Study characteristics.

\begin{tabular}{|c|c|c|c|c|c|c|c|c|}
\hline First author [ref] & Year & Location & Type of study & $\mathbf{N}$ & LNM +, n (\%) & Rectum, n (\%) & Male, n (\%) & $\begin{array}{l}\text { Mean age } \\
\text { (range/ } \pm \text { SD) }\end{array}$ \\
\hline Haggitt [41] & 1985 & Tennessee, USA & Single center & $44^{1} 129^{2}$ & $4(9.1)$ & $42(32.6)^{4}$ & $56(43.4)^{4}$ & $63(29-90)$ \\
\hline Kitamura [28] & 1997 & Kyoto, Japan & Single center & 90 & $7(7.8)$ & $38(42.2)$ & $53(58.9)$ & $\mathrm{u} / \mathrm{k}$ \\
\hline Tsuruta [54] & 2000 & Kurume, Japan & Single center & 77 & $13(16.9)$ & $16(20.8)$ & $56(72.7)$ & $63( \pm 10)$ \\
\hline Oh-e [50] & 2001 & Hiroshima, Japan & Single center & 254 & $35(13.8)$ & $92(36.2)$ & $\mathrm{u} / \mathrm{k}$ & $u / k$ \\
\hline Nascimbeni [49] & 2002 & Rochester, USA & Single center & 353 & $46(13.0)$ & $119(33.7)$ & $204(57.8)$ & $68(36-95)]$ \\
\hline Suzuki [53] & 2003 & Kanagawa, Japan & Single center & 65 & $11(16.9)$ & $23(35.4)$ & $\mathrm{u} / \mathrm{k}$ & $\mathrm{u} / \mathrm{k}$ \\
\hline Egashira [44] & 2004 & Osaka, Japan & Single center & 140 & $13(9.3)$ & $u / k$ & $\mathrm{u} / \mathrm{k}$ & $u / k$ \\
\hline Kitajima [47] & 2004 & Tochigi, Japan & Multicenter & 865 & $87(10.1)$ & $350(40.5)$ & $514(59.4)$ & $63( \pm 11)$ \\
\hline Okabe [51] & 2004 & $\begin{array}{l}\text { New York, USA/ } \\
\text { Tokyo, Japan }\end{array}$ & Multicenter $^{3}$ & 428 & $43(10.0)$ & $176(41.1)$ & $258(60.3)$ & $64(26-89)$ \\
\hline Ueno [42] & 2004 & Saitama, Japan & Single center & $251^{1} 292^{2}$ & $33(13.1)$ & $114(39.0)^{4}$ & $174(59.6)^{4}$ & $62(32-91)$ \\
\hline Yamamoto [38] & 2004 & Tokyo, Japan & Single center & 301 & $19(6.3)$ & $85(28.2)$ & $218(72.4)$ & $\mathrm{u} / \mathrm{k}$ \\
\hline Endreseth [40] & 2005 & $\begin{array}{l}\text { Trondheim, } \\
\text { Norway }\end{array}$ & Multicenter ${ }^{3}$ & $256^{1} 291^{2}$ & $29(11.3)$ & $291(100)^{4}$ & $159(54.6)^{4}$ & $\mathrm{u} / \mathrm{k}$ \\
\hline Wang [55] & 2005 & Taiwan & Single center & 159 & $16(10.1)$ & $75(47.2)$ & $107(67.3)$ & $65(18-89)$ \\
\hline Masaki [48] & 2006 & Tokyo, Japan & Single center & 76 & $5(6.6)$ & $20(26.3)$ & $51(67.1)$ & $68(40-87)$ \\
\hline Ishikawa [46] & 2008 & Tokyo, Japan & $\begin{array}{l}\text { Multicenter, } \\
\text { case-control }\end{array}$ & 71 & $28(39.4)$ & $27(38.0)$ & $48(67.6)$ & $\mathrm{u} / \mathrm{k}$ \\
\hline Rasheed [52] & 2008 & St Marks, UK & Single center & 55 & $7(12.7)$ & $55(100)$ & $33(60.0)$ & $\mathrm{u} / \mathrm{k}$ \\
\hline Ishii [45] & 2009 & Shizuoka, Japan & Single center & 136 & $18(13.2)$ & $38(27.9)$ & $82(60.3)$ & $65(29-91)$ \\
\hline
\end{tabular}

LNM, lymph node metastasis; u/k, unknown. All studies are retrospective, unless stated otherwise.

${ }^{1}$ Cases with available nodal status

2 Total no. of cases.

3 Prospective studies.

${ }^{4}$ All studies are retrospective, unless stated otherwise. 


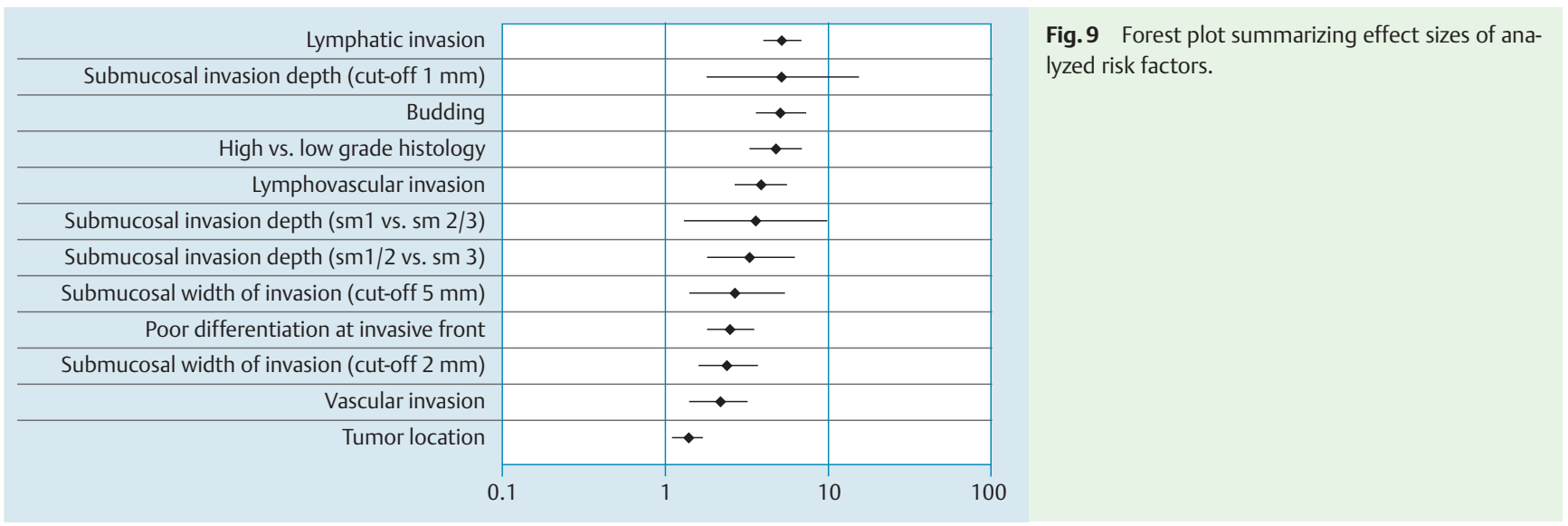

dent effect. It is important to report lymphatic invasion and vascular/venous invasion separately, as vascular invasion is a much weaker predictor of LNM (RR 2.2 [95\%CI 1.4-3.2]). Combining the two factors as lymphovascular invasion logically generates an intermediate relative risk ( $\mathrm{RR} 3.9$ [95\%CI 2.7-5.6]), which is less informative. Additional staining techniques increased interobserver agreement for lymphatic invasion from fair in hematoxylin and eosin $(\mathrm{H} \& \mathrm{E})$-stained slides $(\mathrm{K}=0.30)$ to moderate $(\mathrm{K}=$ 0.56 ) in D2-40-stained slides, and for vascular invasion from considerable for $\mathrm{H} \& \mathrm{E}$ slides $(\mathrm{K}=0.10)$, with marked improvement after EVG staining $(\mathrm{K}=0.48)$ [56].

\section{Tumor budding}

Budding, which is also called "sprouting" or "single cell infiltration," was reported in seven studies $[42,44,46,47,51,54,55]$ totaling 1991 patients. The various authors did not use a uniform definition; however, budding is usually described as foci of isolated cancer cells or clusters of fewer than five cancer cells at the invasive front of the lesion. Ueno et al. [42] regarded five or more of these foci in a microscopic field at $\times 200$ magnification as positive, whereas Ishikawa et al. [46] required more than four foci at a magnification of $\times 400$. Other authors did not provide a cut-off value. Interestingly, the use of various definitions and cut-off values did not result in significant heterogeneity in the meta-analysis $\left(\chi^{2}=7.54, P=0.27, \mathrm{I}^{2}=20 \%\right.$; Table 2$)$. The relative risk was strongly increased in the presence of budding (RR 5.1 [95\%CI $3.6-7.3]$ ), and five out of six studies showed an independent predictive value.

\section{Submucosal invasion depth: qualitative or quantitative measurement}

In this meta-analyses, studies that evaluated submucosal invasion depth for sessile lesions were divided into two groups consisting of the ones applying a qualitative or semi-quantitative definition $[49,52,54,55]$ and those applying a strictly quantitative definition for submucosal invasion [38,42,47,48,50,51]. Although the studies by Masaki et al. [48] and Yamamoto et al. [38] used the designations $s \mathrm{~m} 1, \mathrm{sm} 2$, and $\mathrm{sm} 3$, they were included in the quantitative group because they defined sm levels according to a specific invasion depth in $\mathrm{mm}$. In both groups, submucosal invasion depth was strongly associated with risk of LNM. Increasing semi-quantitatively determined invasion depth was associated with increased risk of LNM ( $\mathrm{sm} 1 / 2$ vs. sm3, RR 3.3 [95\%CI 1.8-6.2]); sm1 vs. sm2/3, RR 3.6 [95\%CI 1.3-9.8]) ( Table 3). However, an independent value was only shown in 1 of 3 multivariable tests (sm1 vs. sm3, Nascimbeni et al. [49]).
In the current meta-analysis there was a significant difference between sm2 and sm3 (RR 2.7 [95\%CI 1.6-4.4]), but only a trend for sm1 vs. sm2 (RR 2.4 [95\%CI 0.9-6.1]; $P=0.08$ ) ( $\bullet$ Fig.e8).

For specimens lacking a muscularis propria layer, quantitative measurement of the invasion depth from the muscularis mucosa to the deepest part of invasion was an alternative. An invasion depth of $\geq 1 \mathrm{~mm}$ into the submucosa showed a strong increase in relative risk for LNM (RR 5.2 [95\%CI 1.8 - 15.4]), and was an independent predictive factor in 2 out of 3 multivariable analyses.

\section{Additional histological factors}

Several additional histological factors were evaluated; however, a meta-analysis was not justified in these cases because of a small number of studies, use of varying definitions and classifications, or lack of relevant data. These factors included submucosal invasion depth according to Haggitt levels for polypoid lesions, tumor size, histological tumor type, presence of inflammatory infiltrate, growth pattern, a cribriform subtype, microvessel density, and macroscopic tumor type.

\section{Discussion \\ $\nabla$}

Published data of 3621 patients with pT1 CRC and available nodal status following radical resection were included in this systematic review. The tumor-related factors that showed the strongest independent predictive value for LNM were lymphatic invasion, budding, poor histological differentiation, and a submucosal invasion depth $\geq 1 \mathrm{~mm}$. As the most important principle for all fit patients with a pT1 CRC is therapy with curative intent, focus should be on selecting patients who have a very low risk and can safely be spared radical surgery.

Several issues need to be resolved before such selection procedures can be performed safely. Standards should be set for histological characteristics in order to improve reproducibility. Appropriate cut-off levels for several factors should be established, and risk stratification models applying a combination of risk factors should be evaluated to establish the optimal combination of predictive factors. Standardization starts with the use of specific definitions. The results for the predictive value of lymphatic invasion illustrate this. Although lymphatic invasion is a strong and reproducible predictive factor for LNM, especially when determined by specific antibody staining [56], the combination with vascular invasion results in an intermediate relative risk, which is not informative enough for clinical treatment decisions. Similarly, histological differentiation is well known for its interobser- 


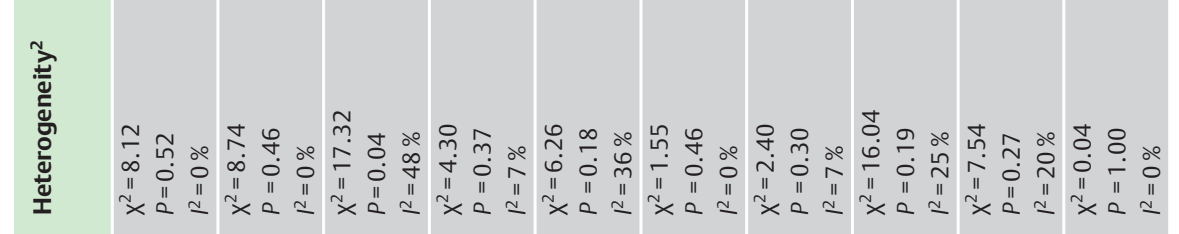

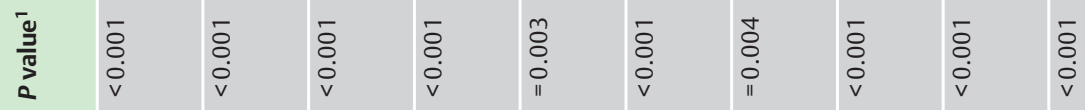

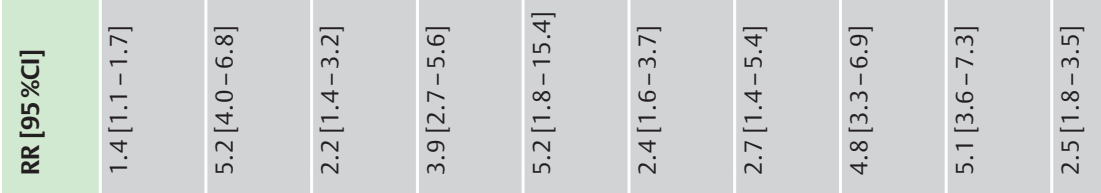

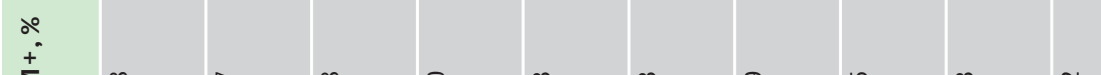

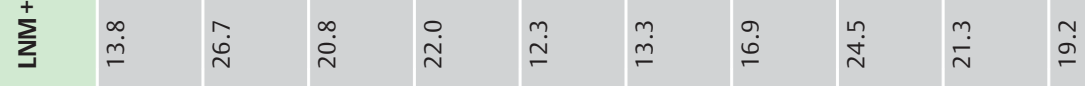

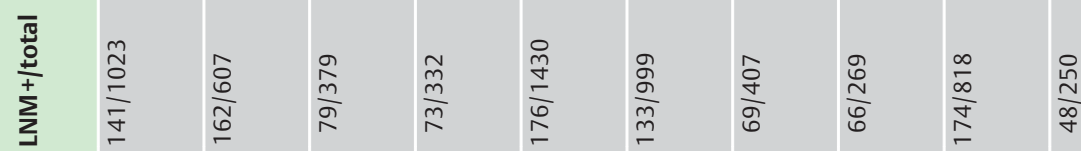

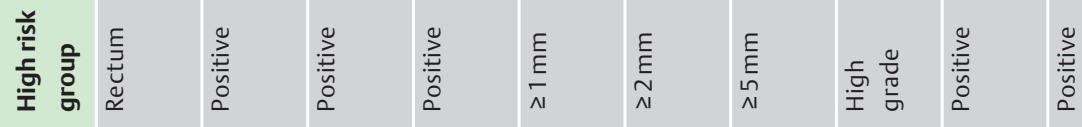

送

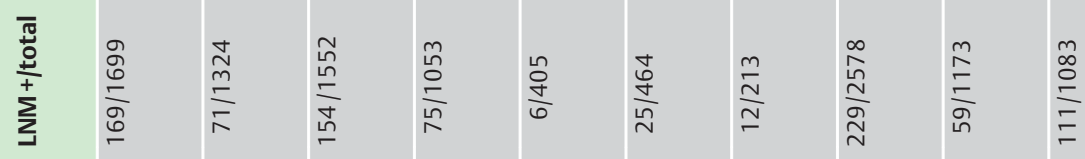

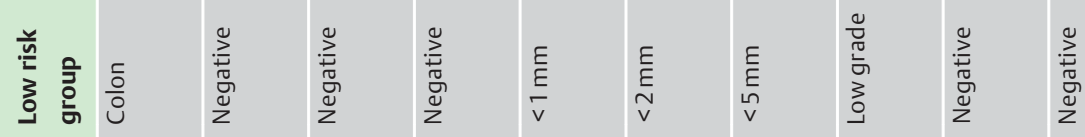

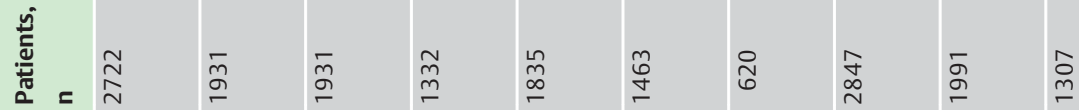

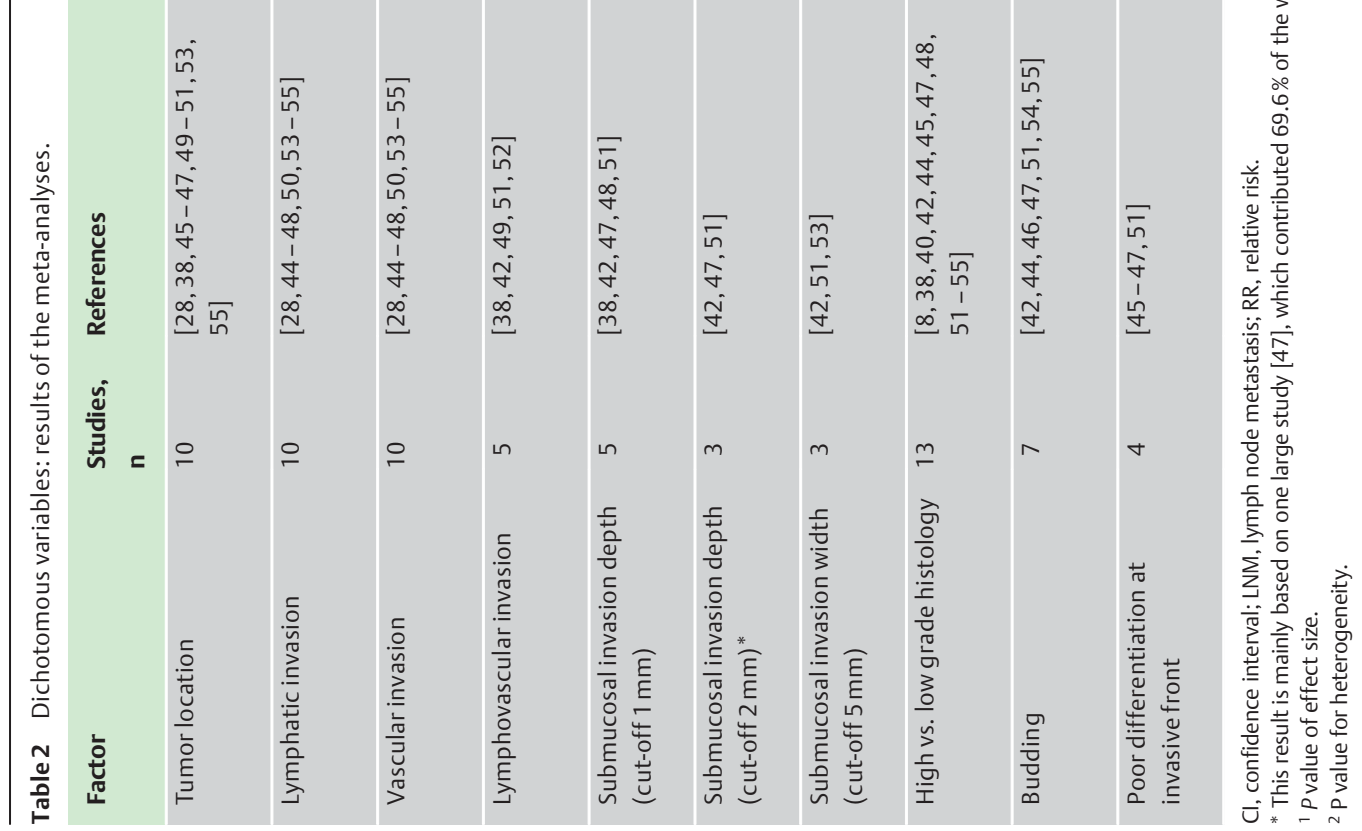


Table 3 Submucosal invasion levels and nodal involvement.

\begin{tabular}{|c|c|c|c|c|c|c|c|c|c|}
\hline Comparison ${ }^{1}$ & $\begin{array}{l}\text { Low risk } \\
\text { group }\end{array}$ & LNM +/total & LNM +, \% & $\begin{array}{l}\text { High risk } \\
\text { group }\end{array}$ & LNM +/total & LNM +, \% & $\mathrm{RR}[95 \% \mathrm{Cl}]$ & $P$ value $^{2}$ & Heterogeneity ${ }^{3}$ \\
\hline $\mathrm{sm} 1$ vs. sm2 & $\mathrm{sm} 1$ & $6 / 174$ & 3.4 & $\mathrm{sm} 2$ & $17 / 200$ & 8.5 & $2.4[0.9-6.1]$ & $=0.08$ & $\begin{array}{l}X^{2}=1.01 \\
P=0.8 \\
I^{2}=0 \%\end{array}$ \\
\hline $\mathrm{sm} 2$ vs. sm 3 & $\mathrm{sm} 2$ & $17 / 200$ & 8.5 & $\mathrm{sm} 3$ & $59 / 261$ & 22.6 & $2.7[1.6-4.4]$ & $<0.001$ & $\begin{array}{l}X^{2}=2.08 \\
P=0.56 \\
I^{2}=0 \%\end{array}$ \\
\hline $\mathrm{sm} 1$ vs. sm 3 & $\mathrm{sm} 1$ & $6 / 174$ & 3.4 & $\mathrm{sm} 3$ & $59 / 261$ & 22.6 & $4.8[1.5-16.2]$ & $=0.01$ & $\begin{array}{l}X^{2}=6.10 \\
P=0.11 \\
I^{2}=51 \%\end{array}$ \\
\hline $\mathrm{sm} 1$ vs. $\mathrm{sm} 2 / 3$ & $\mathrm{sm} 1$ & $6 / 174$ & 3.4 & $\operatorname{sm} 2 / 3$ & $76 / 461$ & 16.5 & $3.6[1.3-9.8]$ & $=0.01$ & $\begin{array}{l}X^{2}=4.40 \\
P=0.22 \\
I^{2}=32 \%\end{array}$ \\
\hline $\mathrm{sm} 1 / 2$ vs. sm 3 & $\mathrm{sm} 1 / 2$ & $23 / 374$ & 6.1 & $\mathrm{sm} 3$ & $59 / 261$ & 22.6 & $3.3[1.8-6.2]$ & $<0.001$ & $\begin{array}{l}X^{2}=4.90 \\
P=0.18 \\
I^{2}=39 \%\end{array}$ \\
\hline
\end{tabular}

$\mathrm{Cl}$, confidence interval; LNM, lymph node metastasis; RR, relative risk.

${ }^{1}$ Data are presented as pooled estimates and relative risks with associated heterogeneity. Data are extracted from four studies (refs. [49,52,54,55]), with a total of 635 patients.

${ }^{2} P$ value of effect size.

${ }^{3} P$ value for heterogeneity.

ver variation $[57,58]$, although the current classification in which low grade and high grade tumors are distinguished has improved reproducibility [59]. In the current study, poor histological differentiation or high grade carcinoma was indeed a strong predictor of LNM, with confirmation in 4 out of 10 multivariable analyses. Budding was a relatively new and not routinely reported risk factor that consistently showed a strong association with the presence of LNM. However, many different definitions were used throughout the literature and there was limited evidence for reproducibility [60]. However, the strong result from the meta-analysis, which lacked significant heterogeneity, indicated that budding, evaluated by any method, is still a powerful marker for LNM.

Establishing cut-off levels is especially important in the determination of submucosal invasion depth. Although semi-quantitative methods have proven to be useful in subsets of tumors in the literature, their value in daily practice might be more limited. Especially in endoscopically resected specimens, the muscularis propria is often missing and the involved proportion of the submucosal layer is therefore hard to estimate. Quantitative measurement of the invasion depth from the muscularis mucosa to the deepest part of invasion is a more feasible method, although the muscularis mucosa may not always be identifiable due to tumor overgrowth [42]. On the other hand, an invasion depth of more than $1 \mathrm{~mm}$ into the submucosa showed a strong increase in relative risk for LNM (RR 5.2 [95\%CI $1.8-15.4]$ ), and was an independent predictive factor in 2 out of 3 multivariable analyses, suggesting it could be a helpful tool for risk stratification. Indeed, assuming the pooled data are representative for clinical practice, a 1-mm cut-off point would assign LNM-positive patients to the high risk group with a sensitivity of $96.7 \%$. However, this would be at the expense of a low specificity (24.1\%) resulting in a high number of patients undergoing unnecessary surgery (false positives). A 1-mm cut-off may therefore not be the optimal method for risk stratification.

As becomes clear from $\bullet$ Table 4, no single predictor discussed in this review allows an optimal selection of low-risk patients by itself, as they are either not sensitive or not specific enough. It therefore seems sensible to investigate the potential of combining risk factors in algorithms in order to identify low risk patients.

Ueno et al. [42] investigated several combinations of risk factors and identified a low risk group, defined by absence of unfavorable grade, lymphovascular invasion, and budding, which contained $55 \%$ of patients and was associated with only $0.7 \%$ nodal involvement. The addition of submucosal depth of invasion $\geq 2$ $\mathrm{mm}$ as a high risk factor eliminated nodal involvement in the low risk group, but also decreased the percentage of low risk patients to $32.3 \%$.

\begin{tabular}{|c|c|c|c|c|}
\hline Factor & Sensitivity, \% & Specificity, \% & PPV, \% & NPV, \% \\
\hline Tumor location in rectum & 45.5 & 63.4 & 13.8 & 90.1 \\
\hline Lymphatic invasion + & 69.5 & 73.8 & 26.7 & 94.6 \\
\hline Vascular invasion + & 33.9 & 82.3 & 20.8 & 90.1 \\
\hline Lymphovascular invasion + & 49.3 & 79.1 & 22.0 & 92.8 \\
\hline Submucosal invasion depth $\geq 1 \mathrm{~mm}$ & 96.7 & 24.1 & 12.3 & 98.5 \\
\hline Submucosal invasion depth $\geq 2 \mathrm{~mm}$ & 84.2 & 33.6 & 13.3 & 94.6 \\
\hline Submucosal width of invasion $\geq 5 \mathrm{~mm}$ & 85.2 & 37.3 & 17.0 & 94.4 \\
\hline High grade histology & 22.4 & 92.0 & 24.5 & 91.1 \\
\hline Budding & 74.7 & 63.4 & 21.3 & 95.0 \\
\hline Poor differentiation at invasive front & 30.2 & 82.8 & 19.2 & 89.8 \\
\hline $\mathrm{sm} 2 / 3$ (vs. sm 1 ) & 92.7 & 30.4 & 16.8 & 96.6 \\
\hline $\operatorname{sm} 3$ (vs. sm1/2) & 72.0 & 63.5 & 22.6 & 93.9 \\
\hline
\end{tabular}

Table 4 Nodal involvement: Sensitivity, specificity, positive predictive value, and negative predictive value for the identified histological risk factors. 
In a paper by Nakadoi et al. [61], the authors employed the high risk factors described in the 2010 guidelines of the Japanese Society for Cancer of the Colon and Rectum [36], which are very similar to the ones used by Ueno et al. [42]. They found that when a tumor was low risk (well/moderately differentiated, no vascular invasion or tumor budding) the incidence of LNM was only $1.2 \%$ with $49.9 \%$ of patients assigned to the low risk group. A limitation of the current study is that it was not possible to investigate combinations of factors. Another inevitable source of bias is that this systematic review only included tumors from patients who underwent radical surgery. The population of patients with early $\mathrm{T} 1$ cancers that were removed by polypectomy without radical surgery was not included in the current analysis because the definitive lymph node status was not available. This omission is especially relevant for pedunculated lesions, which undergo local excision more frequently [42], and are known to have a low risk of LNM [47]. Future studies should take this into account.

Furthermore, the majority of papers included in this study originated from Asian countries in which pathological work-up may be more extensive, and which might influence results. Validation of the currently identified risk factors in Western populations is necessary.

In conclusion, several factors can be identified in pT1 tumors that predict the presence of LNM. Lymphatic invasion, budding, submucosal invasion depth $\geq 1 \mathrm{~mm}$, and poor histological differentiation are the strongest predictors of LNM that also show a consistent independent predictive value in multivariable analyses. Their absence indicates a low risk of LNM and may justify a conservative policy. Future studies should investigate all of the above-mentioned factors and aim to standardize the detection of these powerful markers, preferably using immunohistochemical staining techniques. These recommendations may lead to the development of a validated model incorporating various risk factors for the prediction of LNM, which may help to select patients who can be spared radical resection, and such a model may thereby prevent unnecessary surgery without compromising oncological safety.

\section{Competing interests: None}

\section{Institutions}

${ }^{1}$ Department of Pathology, Radboud University Nijmegen Medical Center, Nijmegen, The Netherlands,

2 Department of Epidemiology, Biostatistics and HTA, Radboud University Nijmegen Medical Center, Nijmegen, The Netherlands,

${ }^{3}$ Department of Surgery, Radboud University Nijmegen Medical Center, Nijmegen, The Netherlands

${ }^{4}$ Department of Colorectal Surgery, Churchill Hospital, Oxford University

Hospitals, Oxford, United Kingdom

\section{References}

1 Hardcastle JD, Chamberlain JO, Robinson MH et al. Randomised controlled trial of faecal-occult-blood screening for colorectal cancer. Lancet 1996; 348: 1472 - 1477

2 Kewenter J, Brevinge H, Engaras B et al. Results of screening, rescreening, and follow-up in a prospective randomized study for detection of colorectal cancer by fecal occult blood testing. Results for 68,308 subjects. Scand J Gastroenterol 1994; 29: 468-473

3 Kronborg 0 , Fenger $C$, Olsen $J$ et al. Randomised study of screening for colorectal cancer with faecal-occult-blood test. Lancet 1996; 348 : $1467-1471$

4 Mandel JS, Bond JH, Church TR et al. Reducing mortality from colorectal cancer by screening for fecal occult blood. Minnesota Colon Cancer Control Study. N Engl J Med 1993; 328: 1365 -1371
5 Paimela $\mathrm{H}$, Malila $\mathrm{N}$, Palva $\mathrm{T}$ et al. Early detection of colorectal cancer with faecal occult blood test screening. Br J Surg 2010; 97: 1567-1571 6 Smart CR. Screening and early diagnosis. Cancer 1992; 70: 1246-1251

7 Takeuchi Y, Uedo N, Ishihara $R$ et al. Efficacy of an endo-knife with a water-jet function (Flushknife) for endoscopic submucosal dissection of superficial colorectal neoplasms. Am J Gastroenterol 2010; 105: $314-322$

8 Winde G, Nottberg $H$, Keller $R$ et al. Surgical cure for early rectal carcinomas (T1). Transanal endoscopic microsurgery vs. anterior resection. Dis Colon Rectum 1996; 39: 969-976

9 Langer C, Liersch T, Suss M et al. Surgical cure for early rectal carcinoma and large adenoma: transanal endoscopic microsurgery (using ultrasound or electrosurgery) compared to conventional local and radical resection. Int J Colorectal Dis 2003; 18: 222 -229

10 Lee $W$, Lee $D$, Choi $S$ et al. Transanal endoscopic microsurgery and radical surgery for T1 and T2 rectal cancer. Surg Endosc 2003; 17: 1283 1287

$11 W u Y, W u Y Y$, Li S et al. TEM and conventional rectal surgery for T1 rectal cancer: a meta-analysis. Hepatogastroenterology 2011; 58: $364-$ 368

12 Tada M, Inoue H, Yabata E et al. Feasibility of the transparent cap-fitted colonoscope for screening and mucosal resection. Dis Colon Rectum 1997; 40: 618-621

13 Lee EJ, Lee JB, Lee SH et al. Endoscopic treatment of large colorectal tumors: comparison of endoscopic mucosal resection, endoscopic mucosal resection-precutting, and endoscopic submucosal dissection. Surg Endosc 2012; 26: 2220-2230

14 Iversen LH, Nielsen $H$, Pedersen $L$ et al. Seasonal variation in short-term mortality after surgery for colorectal cancer? Colorectal Dis 2010; 12 : e31-36

15 Paulson EC, Mitra N, Sonnad S et al. National Cancer Institute designation predicts improved outcomes in colorectal cancer surgery. Ann Surg 2008; 248: 675-686

16 Vironen $J H$, Kairaluoma $M$, Aalto AM et al. Impact of functional results on quality of life after rectal cancer surgery. Dis Colon Rectum 2006; 49: $568-578$

17 Balch GC, De Meo A, Guillem JG. Modern management of rectal cancer: a 2006 update. World J Gastroenterol 2006; 12: 3186-3195

18 Engel J, Kerr J, Schlesinger-Raab A et al. Quality of life in rectal cancer patients: a four-year prospective study. Ann Surg 2003; 238: 203-213

19 Chatwin NA, Ribordy M, Givel JC. Clinical outcomes and quality of life after low anterior resection for rectal cancer. Eur J Surg 2002; 168: 297-301

20 Braga M, Frasson M, Zuliani Wet al. Randomized clinical trial of laparoscopic versus open left colonic resection. Br J Surg 2010; 97: 1180 1186

21 Tanaka S, Oka S, Kaneko I et al. Endoscopic submucosal dissection for colorectal neoplasia: possibility of standardization. Gastrointest Endosc 2007; 66: 100-107

22 Cahill RA, Leroy J, Marescaux J. Localized resection for colon cancer. Surg Oncol 2009; 18: $334-342$

23 Kato H, Haga S, Endo S et al. Lifting of lesions during endoscopic mucosal resection (EMR) of early colorectal cancer: implications for the assessment of resectability. Endoscopy 2001; 33: 568 - 573

24 Mellgren A, Sirivongs $P$, Rothenberger $D A$ et al. Is local excision adequate therapy for early rectal cancer? Dis Colon Rectum 2000; 43: 1064 1071 ; discussion 1071-1064

25 Tanaka S, Haruma K, Teixeira CR et al. Endoscopic treatment of submucosal invasive colorectal carcinoma with special reference to risk factors for lymph node metastasis. J Gastroenterol 1995; 30: 710 - 717

26 Kobayashi H, Mochizuki H, Morita $T$ et al. Characteristics of recurrence after curative resection for $\mathrm{T} 1$ colorectal cancer: Japanese multicenter study. J Gastroenterol 2011; 46: 203-211

27 Coverlizza S, Risio M, Ferrari A et al. Colorectal adenomas containing invasive carcinoma. Pathologic assessment of lymph node metastatic potential. Cancer 1989; 64: 1937-1947

28 Kitamura K, Taniguchi H, Yamaguchi T et al. Clinical outcome of surgical treatment for invasive early colorectal cancer in Japan. Hepatogastroenterology 1997; 44: 108-115

29 Graham RA, Garnsey L, Jessup JM. Local excision of rectal carcinoma. Am J Surg 1990; 160: 306-312

30 Brodsky JT, Richard GK, Cohen AM et al. Variables correlated with the risk of lymph node metastasis in early rectal cancer. Cancer 1992; 69: $322-326$ 
31 Blumberg D, Paty PB, Guillem JG et al. All patients with small intramural rectal cancers are at risk for lymph node metastasis. Dis Colon Rectum 1999; 42: $881-885$

32 Kikuchi R, Takano M, Takagi K et al. Management of early invasive colorectal cancer. Risk of recurrence and clinical guidelines. Dis Colon Rectum 1995; 38: 1286 - 1295

33 National Cancer Institute. Rectal Cancer Treatment (PDQ ${ }^{\circledR}$ ). 2011: Available from: http://www.cancer.gov/cancertopics/pdq/treatment/ rectal/HealthProfessional Accessed: 17 February 2012

34 The Association of Coloproctology of Great Britain and Ireland. Guidelines for the management of colorectal cancer. edn 2007; 3rd: Available from: http://www.acpgbi.org.uk/wp-content/uploads/2007-CCManagement-Guidelines.pdf Accessed: 17 February 2012

35 Oncoline. Cancer clinical practice guidelines. Rectal cancer. Version: 2.0 2008: Available from: http://oncoline.nl/index.php?pagina=/richtlijn/item/pagina.php\&richtlijn_id=615 Accessed: 17 February 2012

36 Japanese Society for Cancer of the Colon and Rectum. Japanese guidelines for the treatment of colorectal carcinoma. Tokyo: Kanehara Shuppan Co; 2010: $42-43$

37 Kudo S. Endoscopic mucosal resection of flat and depressed types of early colorectal cancer. Endoscopy 1993; 25: 455-461

38 Yamamoto $S$, Watanabe $M$, Hasegawa $H$ et al. The risk of lymph node metastasis in T1 colorectal carcinoma. Hepatogastroenterology 2004; 51: $998-1000$

39 Sakuragi M, Togashi K, Konishi $F$ et al. Predictive factors for lymph node metastasis in T1 stage colorectal carcinomas. Dis Colon Rectum 2003; 46: $1626-1632$

40 Endreseth BH, Myrvold HE, Romundstad $P$ et al. Transanal excision vs. major surgery for T1 rectal cancer. Dis Colon Rectum 2005; 48: $1380-1388$

41 Haggitt RC, Glotzbach RE, Soffer EE et al. Prognostic factors in colorectal carcinomas arising in adenomas: implications for lesions removed by endoscopic polypectomy. Gastroenterology 1985; 89: 328-336

42 Ueno H, Mochizuki H, Hashiguchi Yet al. Risk factors for an adverse outcome in early invasive colorectal carcinoma. Gastroenterology 2004; 127: $385-394$

43 Son HJ, Song SY, Lee WY et al. Characteristics of early colorectal carcinoma with lymph node metastatic disease. Hepatogastroenterology 2008; 55: $1293-1297$

44 Egashira Y, Yoshida T, Hirata I et al. Analysis of pathological risk factors for lymph node metastasis of submucosal invasive colon cancer. Mod Pathol 2004; 17: 503-511

45 Ishii $M$, Ota $M$, Saito $S$ et al. Lymphatic vessel invasion detected by monoclonal antibody D2-40 as a predictor of lymph node metastasis in T1 colorectal cancer. Int J Colorectal Dis 2009; 24: 1069-1074

46 Ishikawa Y, Akishima-Fukasawa $Y$, Ito $K$ et al. Histopathologic determinants of regional lymph node metastasis in early colorectal cancer. Cancer 2008; 112: 924-933
47 Kitajima K, Fujimori T, Fujii S et al. Correlations between lymph node metastasis and depth of submucosal invasion in submucosal invasive colorectal carcinoma: a Japanese collaborative study. J Gastroenterol 2004; 39: 534-543

48 Masaki T, Matsuoka H, Sugiyama M et al. Actual number of tumor budding as a new tool for the individualization of treatment of T1 colorectal carcinomas. J Gastroenterol Hepatol 2006; 21: 1115-1121

49 Nascimbeni R, Burgart LJ, Nivatvongs $S$ et al. Risk of lymph node metastasis in T1 carcinoma of the colon and rectum. Dis Colon Rectum 2002; 45: $200-206$

50 Oh-e H, Tanaka S, Kitadai Yet al. Angiogenesis at the site of deepest penetration predicts lymph node metastasis of submucosal colorectal cancer. Dis Colon Rectum 2001; 44: 1129-1136

51 Okabe S, Shia J, Nash G et al. Lymph node metastasis in T1 adenocarcinoma of the colon and rectum. J Gastrointest Surg 2004; 8: 1032 1039 ; discussion 1039-1040

52 Rasheed S, Bowley DM, Aziz O et al. Can depth of tumour invasion predict lymph node positivity in patients undergoing resection for early rectal cancer? A comparative study between T1 and T2 cancers Colorectal Dis 2008; 10: 231-238

53 Suzuki T, Sadahiro S, Mukoyama S et al. Risk of lymph node and distant metastases in patients with early invasive colorectal cancer classified as Haggitt's level 4 invasion: image analysis of submucosal layer invasion. Dis Colon Rectum 2003; 46: 203-208

54 Tsuruta O, Tsuji Y, Kawano $H$ et al. Indication for endoscopic resection of submucosal colorectal carcinoma: special reference to lymph node metastasis. Diagn Ther Endosc 2000; 6: 101 - 109

55 Wang HS, Liang WY, Lin TC et al. Curative resection of T1 colorectal carcinoma: risk of lymph node metastasis and long-term prognosis. Dis Colon Rectum 2005; 48: 1182 - 1192

56 Suzuki A, Togashi K, Nokubi M et al. Evaluation of venous invasion by Elastica van Gieson stain and tumor budding predicts local and distant metastases in patients with T1 stage colorectal cancer. Am J Surg Pathol 2009; 33: $1601-1607$

57 Blenkinsopp WK, Stewart-Brown S, Blesovsky L et al. Histopathology reporting in large bowel cancer. J Clin Pathol 1981; 34: 509-513

58 Thomas GD, Dixon MF, Smeeton NC et al. Observer variation in the histological grading of rectal carcinoma. J Clin Pathol 1983; 36: 385-391

59 Bosman FT. World Health Organization, International Agency for Research on Cancer. WHO classification of tumours of the digestive system. 4th: edn. Lyon: International Agency for Research on Cancer; 2010

60 Vieth M, Quirke P, Lambert R et al. Annex to Quirke et al. Quality assurance in pathology in colorectal cancer screening and diagnosis: annotations of colorectal lesions. Virchows Arch 2011; 458: 21 - 30

61 Nakadoi K, Tanaka S, Kanao H et al. Management of T1 colorectal carcinoma with special reference to criteria for curative endoscopic resection. J Gastroenterol Hepatol 2012; 27: 1057-1062

Figure e2-e8, Table e5

online content viewable at: www.thieme-connect.de 